\title{
Limitations in Electronic Prescribing in Sri Lankan context: A qualitative study
}

\author{
M.H.B. Ariyaratne \\ Ministry of Health, Nutrition and Indigenous Medicine, Sri Lanka \\ buddhika.ari@gmail.com \\ https://orcid.org/0000-0002-1798-6203 \\ ABSTRACT
}

\begin{abstract}
Introduction: The process of creating medical prescriptions by hand using a pen and paper is influenced by the knowledge, experience, guidelines and regulations. When the same activity is attempted using e-prescription software, user interface and system design limitations can impose additional restrictions. Such additional restrictions limit the freedom to adhere to all possible patterns found in paper-based prescriptions disturbing the workflow and the outputs. The objective of the research was to identify the limitations in e-prescribing and to propose improvements.
\end{abstract}

Methods: A multistage integrated evaluation was conducted to identify the limitations. The first stage was the identification of the work process of paper-based prescription generation and patterns of the outputs. The second stage consisted of an anonymous online questionnaire to capture the usability data of each identified case. The third stage was a semi-structured interview among the Sri Lankan electronic prescribers to capture the capability of electronic prescribing to address the identified use cases.

Results: The data model for the Dictionary of Medicines and Devices $(d m+d)$ of National Health Services, UK was used to model seven different use cases which were explained by activity diagrams, component diagrams and test cases at the first stage. During the second stage of the online questionnaire, all the use cases identified were found to be used during the generation of paper-based prescriptions. The thematic analysis of semistructured interviews revealed that e-prescribing fails in some identified use cases.

Conclusions: Some Electronic Health Record Systems used in Sri Lanka are currently not fit enough to cater to certain identified use cases of prescription generation. The limitations need to be recognized and corrected before the development of unintentional trends among Sri Lankan e-prescribers.

Keywords: e-prescribing, dmd+d, 